\title{
LA VENERACIÓN DE MONTAÑAS EN LOS ANDES PREINCAICOS: EL CASO DE ÑAWINPUKYO (AYACUCHO, PERÚ) EN EL PERÍODO INTERMEDIO TEMPRANO
}

\author{
MOUNTAIN WORSHIP IN THE PRE-INCA ANDES: \\ THE CASE OF NAWINPUKYO (AYACUCHO, PERU) IN THE EARLY \\ INTERMEDIATE PERIOD
}

\begin{abstract}
Juan B. Leoni*
La veneración de montañas juega un papel central en la cosmología y religión de las sociedades andinas tradicionales, como lo demuestran numerosos estudios etnográficos, documentos etnohistóricos y restos arqueológicos de altura. Se asume generalmente que las creencias y prácticas relacionadas con la veneración de montañas tienen raíces temporales más profundas, remontándose a siglos o milenios antes de los incas, aunque las formas que esta veneración tomaba y su rol sociopolítico no son tan bien conocidas. En este trabajo se presenta un caso arqueológico que demuestra que la veneración de montañas formaba parte central de la religión de los habitantes de Ñawinpukyo (valle de Ayacucho, Perú) durante el período Intermedio Temprano (ca. 0-550/600 d.C.). Se argumenta que las ceremonias en que se honraba a una deidad de montaña principal constituían un importante mecanismo integrador de la comunidad local, y que posiblemente tenían también un papel importante a nivel regional.

Palabras claves: veneración de montañas, arquitectura ceremonial, período preinca, Ayacucho.

Mountain worship plays a central role in the cosmology and religion of traditional Andean societies, as numerous ethnographic studies, ethnohistorical sources, and high altitude archaeological remains show. It is generally assumed that beliefs and practices associated with mountain worship have deep temporal roots, dating back to centuries or even millennia before the Incas. However, the forms this veneration took and its socio-political role are not so well-known. In this paper I present an archaeological case that shows that mountain worship was a key part of the religion of the inhabitants of the Nawinpukyo site (Ayacucho Valley, Peru) during the Early Intermediate Period (ca. A.D. 0-550/600). I argue that the ceremonies meant to honor a main regional mountain deity constituted an important integrative mechanism for the local community, possibly playing an integrative role on the broader regional level as well.
\end{abstract}

Key words: Mountain worship, ceremonial architecture, pre-inca period, Ayacucho.

El importante papel de las montañas en la cosmología de las sociedades andinas está bien documentado etnográfica y etnohistóricamente. Las evidencias arqueológicas son también abundantes para el período incaico, sobre todo, en la forma de santuarios, sacrificios y ofrendas a gran altura. Asimismo, se asume generalmente que la veneración de montañas forma parte de un núcleo de creencias religiosas y concepciones cosmológicas tradicionales de las sociedades andinas que tendría sus raíces en tiempos muy anteriores a los incas. Sin embargo, no parece que los santuarios de altura similares a los de los incas hayan sido muy comunes, indicando que el culto de las montañas tenía aspectos distintos en épocas más tempranas. En este trabajo se presenta un caso particular que documenta arqueológicamente aspectos de la hasta ahora casi desconocida veneración de montañas preincaica. Se trata de un pequeño complejo ceremonial en el sitio de Ñawinpukyo (Ayacucho, Perú), que data del período Intermedio Temprano (ca. 0-550/600 d.C.) y que incluye un edificio circular claramente relacionado con una montaña de reconocido valor simbólico y religioso. Se discute la evidencia arqueológica hallada, utilizando analogías etnográficas para ilustrar similitudes formales que pueden servir para interpretar algunos de los contextos arqueológicos hallados en dicho complejo. Se evalúa también el rol jugado por las posibles ceremonias relacionadas con la veneración de montañas en la integración de la comunidad tanto a nivel local como regional.

\footnotetext{
* Escuela de Antropología, Facultad de Humanidades y Artes, Universidad Nacional de Rosario. Entre Ríos 758, (2000), Rosario, Argentina. jbleoni@hotmail.com
} 


\section{Los Dioses de las Montañas en la Cosmología Andina}

La importancia de los dioses de las montañas, conocidos generalmente como apus, wamanis o awkillu en distintas regiones de Perú, y machula, achachila o mallku en distintas partes de Bolivia, en la cosmología de las sociedades andinas posthispánicas es bien conocida. Varios estudios etnográficos han documentado las creencias religiosas asociadas con las montañas que existen en los Andes (Allen 2002; Anders 1986; Bastien 1985; Bolin 1998; Favre 1967; Fernández Juárez 1997; Flannery et al. 1989; Isbell 1978; Martínez 1983; Morissette y Racine 1973; Reinhard 1985). De acuerdo con estos estudios, las deidades residen en cerros nevados, promontorios, colinas, cuevas y lagunas de montaña, así como en otros rasgos topográficos menores, y se considera en general que ejercen una gran influencia sobre las comunidades humanas. Controlan los fenómenos meteorológicos y se las considera dueñas de animales y plantas, salvajes y domesticadas. Así, distintos rituales tienen como finalidad propiciar a estas deidades de forma de asegurar la reproducción exitosa de animales, cultivos y, en última instancia, de los seres humanos mismos. Sin embargo, estas deidades también pueden ejercer una influencia negativa, infligiendo daños y castigos en la forma de enfermedades, accidentes e infertilidad tanto de los seres humanos como de los animales y plantas, si son ofendidas o no se las propicia adecuadamente.

Los apus o wamanis se organizan en una jerarquía piramidal en la cual las deidades se sitúan de acuerdo a su importancia. A cada una de las deidades le corresponden territorios sobre los cuales ejercen su influencia, correspondiendo áreas geográficas más extensas a aquellas deidades ubicadas en lo más alto de la jerarquía. En esta organización jerárquica los cerros nevados más altos, visibles desde grandes distancias, comúnmente ejercen su poder e influencia sobre extensiones geográficas mayores, y son, en consecuencia, venerados por grandes número de personas extendidas sobre amplios territorios (Allen 2002:27-28; Anders 1986:762-765; Favre 1967:122; Isbell 1978:59; Morissette y Racine 1973:171). Entonces, los wamanis no sólo se caracterizan por poseer poderes sobrenaturales, sino que también pueden servir como el foco en torno al cual se construyen identidades comunitarias y supracomunitarias. Se- gún Anders (1986:763), “los wamanis pueden ser invocados para dar unidad a territorios de mayor o menor tamaño dependiendo del contexto o la necesidad" [traducido por el autor], operando como símbolos de identidad para grupos sociales de variado nivel de inclusión y extensión geográfica (Reinhard 1985:310). Así, los cerros altos más visibles proveen un foco común para una integración regional más amplia, promoviendo identidades sociales que se reproducen a través de la participación en ceremonias dedicadas a honrar a estas deidades principales (Allen 2002:85).

La estructura jerárquica de los wamanis en Ayacucho ${ }^{1}$ ha sido bien estudiada por varios investigadores (Anders 1986:765-767; Isbell 1978:151; Morissette y Racine 1973:171). Estos estudios han señalado claramente la existencia de tres o cuatro deidades principales, o hatun wamani, que se identifican con cerros nevados. De éstos, el más importante es el cerro Rasuwillka ${ }^{2}$, que se localiza en el flanco norte del valle de Ayacucho (Figura 1), alcanzando una altura máxima de $4.954 \mathrm{msm}$. Esta montaña ejerce su influencia en la región, encontrándose los restantes wamanis subordinados a ella (Morissette y Racine 1973:171).

Las fuentes etnohistóricas señalan que el culto de las montañas tenía gran importancia entre los habitantes del área andina entre los siglos XVI y XVIII, y en especial en tiempos de los incas (Besom 2000; Reinhard 1985). Las ceremonias relacionadas con los cerros constituían una parte importante de la religión del estado incaico, tal como lo atestiguan no sólo los documentos etnohistóricos, sino también los restos arqueológicos hallados en las cimas y laderas de altas montañas (Besom 2000; Reinhard 1985; Schobinger y Cerutti 2001). Según Reinhard (1985:306), las montañas eran veneradas tanto por los incas como por las poblaciones locales incorporadas a su imperio, constituyendo deidades relacionadas con cultos de fertilidad y veneradas para asegurar el suministro de agua. La veneración de montañas estaba, al parecer, profundamente interrelacionada con otras prácticas religiosas de central importancia, tales como los ritos de fertilidad y el culto a los ancestros, como parte de complejos sistemas cosmológicos (Reinhard 1985:309). Sin embargo, no parece existir entre los cronistas un acuerdo generalizado acerca de los significados simbólicos asociados con las montañas. Así, las montañas 


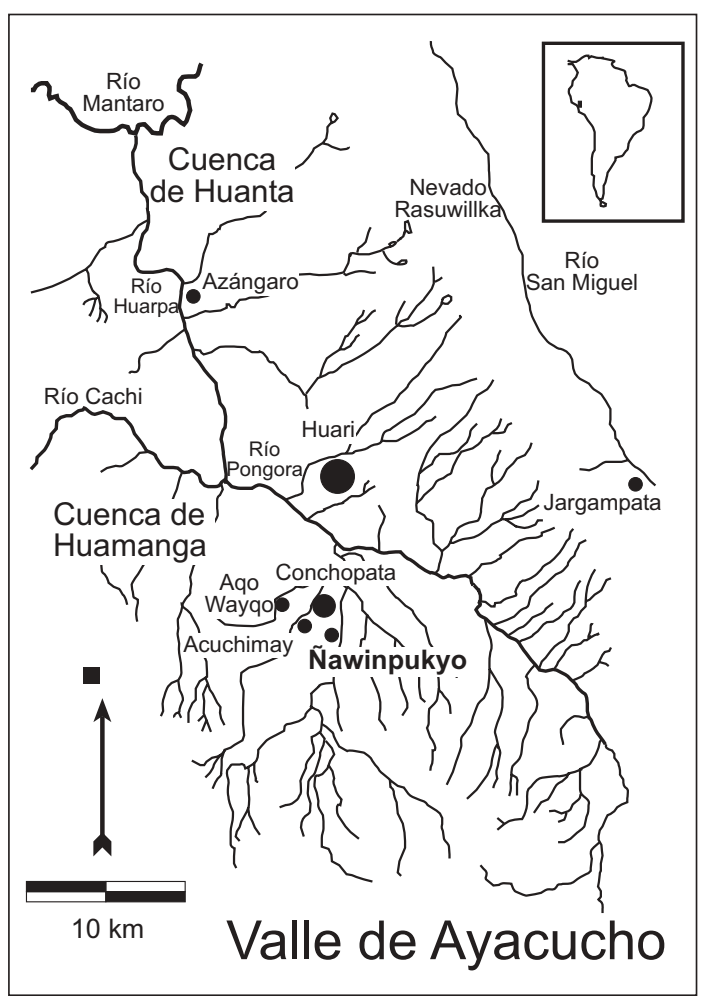

Figura 1. Ñawinpukyo y otros sitios arqueológicos importantes del valle de Ayacucho (redibujado en base a Isbell 2001: Figura 3). Map of the Ayacucho Valley showing location of Nawinpukyo and other major archaeological sites (redrawn from Isbell 2001: Figure 3).

podían ser consideradas alternativamente como huacas o lugares imbuidos con poder sagrado, manifestaciones físicas de seres divinos, restos petrificados de personajes míticos, residencias de deidades y lugares de origen de ciertos grupos étnicos, o bien, conjugar varias de estas categorías al mismo tiempo (Besom 2000:161). Es interesante destacar, como Anders (1986:780-781) señalara, que Guamán Poma de Ayala (1980:193 [1615]) mencionó al nevado Rasuwillka en una lista de las principales huacas del Chinchaysuyu, lo que sugiere que la importancia religiosa de esta montaña registrada etnográficamente puede tener raíces temporales más profundas, rastreables con certeza, por lo menos, a tiempos prehispánicos tardíos.

La importancia de la veneración de montañas para los incas encuentra su máxima expresión material en la proliferación de santuarios de altura que acompaña a la expansión del imperio, especialmente en los Andes meridionales (Besom 2000; Reinhard 1985; Schobinger y Cerutti 2001). La exten- dida construcción de santuarios de altura habría formado parte de una estrategia sistemática de dominación ritual que permitió a los incas apropiarse de las huacas o deidades regionales de los pueblos conquistados, consiguiendo de esta manera un mayor control político sobre las poblaciones incorporadas a su imperio (Besom 2000; Reinhard 1985; Schobinger y Cerutti 2001). Es interesante destacar que, si bien las montañas habrían tenido valor simbólico y religioso para los grupos preincas, la construcción de santuarios sobre las cumbres no habría constituido una parte central de las prácticas de veneración de las montañas. Las formas en que se manifestaban arqueológicamente estas creencias en tiempos preincaicos son entonces más difíciles de discernir.

Varios investigadores han planteado la posibilidad de la existencia de creencias y prácticas religiosas vinculadas con el culto a las montañas en tiempos anteriores a los incas, y variados restos arqueológicos andinos han sido interpretados en función de esta hipótesis (Anders 1986:792-805; Glowacki y Malpass 2003:439-441; Kolata y Ponce Sanginés 1992; Reinhard 1992; entre otros). Según Reinhard (1985:309), la creencia en los dioses de las montañas es claramente anterior a los incas y se hallaba ampliamente extendida a través del área andina (ver también Isbell 1978:214). En su opinión, los dioses de las montañas eran venerados, por ejemplo, en la cuenca del río Nazca mucho antes de la llegada de los españoles, y ha sugerido que los geoglifos de Nazca eran parte integral de un culto que tenía al agua y las montañas como elementos principales (Reinhard 1992; ver también Silverman y Proulx 2002:205-209). De manera similar, Glowacki y Malpass (2003:439-441) argumentan que varios sitios arqueológicos Huari del Horizonte Medio (ca. 550/600-1000 d.C.) fueron intencionalmente construidos en una estrecha asociación física y simbólica con montañas y otros elementos topográficos de valor religioso, conformando un paisaje sagrado que buscaba asegurar el control simbólico de las diversas huacas regionales asociadas con el suministro del agua.

Una interpretación particularmente relevante para el caso que aquí se presenta fue desarrollada por Anders (1986:782-788), afirmando que el culto a las montañas, reportado etnográficamente en la región de Ayacucho, se encontraba ya constituido en el período Intermedio Tardío (ca. 1000-1470 d.C.), sirviendo como base de la organización te- 
rritorial y política de la confederación Chanka. Además, Anders (1986:792-811) llevó su argumento aun más lejos, aduciendo que el patrón identificado para los tiempos Chanka tenía en realidad sus raíces en el Horizonte Medio. Según esta visión, la jerarquía de los wamanis principales de Ayacucho habría sido la base sobre la cual se organizó espacialmente la administración del imperio Huari, conformando un sistema regional cuatripartito en el que cada uno de los wamanis principales presidía su correspondiente sección de la administración estatal.

Muchos de estos estudios, sin embargo, asumen en mayor o menor medida una continuidad cultural ininterrumpida, y la interpretación de los restos arqueológicos se basa sobre todo en la proyección al pasado prehispánico de analogías etnográficas y etnohistóricas. Transformaciones culturales significativas, tanto en tiempos prehispánicos como posthispánicos, podrían haber introducido cambios en las creencias de las sociedades andinas, así como ciertamente ocurrió con sus formas de organización política, económica y social, planteando límites al uso directo de la analogía etnográfica en la arqueología andina (Isbell 1997:124135). Como señalara Isbell (1997:131), la activa persecución, por parte de las autoridades eclesiásticas y civiles coloniales de las prácticas religiosas relacionadas con la veneración de las momias de los ancestros, podría haber desplazado el foco de la religión de los grupos andinos hacia el culto de los dioses de los cerros y de los santos católicos. La notable preeminencia del culto de las montañas en los estudios etnográficos y documentos etnohistóricos tardíos podría así responder, al menos en cierto grado, a procesos históricos de cambio, más que a su pertenencia a un núcleo esencial de creencias tradicionales andinas. Aún aceptando estos planteos, sin embargo, parece claro que ciertos aspectos relacionados con el culto de las montañas bien pudieron haber existido en tiempos prehispánicos más tempranos, aunque no necesariamente iguales a las prácticas reportadas etnográfica y etnohistóricamente. En las páginas que siguen se presentan evidencias materiales del sitio de Ñawinpukyo (Ayacucho, Perú) que sugieren que la veneración de montañas era un aspecto central de la religión de la sociedad Huarpa del período Intermedio Temprano.

\section{El Sitio de Ñawinpukyo}

El sitio de Ñawinpukyo se ubica sobre una colina, a unos $5 \mathrm{~km}$ al sudeste del centro de la actual ciudad de Ayacucho (Figura 1). La colina forma parte del flanco sur del valle y alcanza una altura máxima de $3.007 \mathrm{msm}$. Si bien se encuentran restos arqueológicos en las laderas y parte baja de la colina (Cabrera 1998; Machaca 1997), la concentración más densa se ubica directamente sobre la cima (Leoni 2004; Lumbreras 1974).

La cima de la colina es una planicie suavemente ondulada de unos $500 \mathrm{~m}$ de largo por $200 \mathrm{~m}$ de ancho. En ella se encuentran amplias áreas abiertas que se alternan con densas concentraciones de arquitectura arqueológica en ruinas. Las investigaciones realizadas en 2001 se concentraron en las partes central y este de la cima, y a través de una combinación de limpieza de vegetación y escombros, definición de cabeceras de muro y excavación, se consiguió exponer un área estimada en 650 $\mathrm{m}^{2}$, definiéndose 32 cuartos o estructuras (denominados "Espacios Arquitectónicos") de los cuales 26 fueron total o parcialmente excavados. Las investigaciones revelaron una secuencia ocupacional que se extiende desde el período Intermedio Temprano (ocupación Huarpa del sitio), hasta finales del Horizonte Medio (ocupación Huari de la colina) (Leoni 2004). La discusión presentada aquí se centra en la ocupación Huarpa y, en especial, en la interpretación de un conjunto de edificios y contextos de índole ceremonial.

\section{Ñawinpukyo en el Período Intermedio Temprano}

El período Intermedio Temprano en Ayacucho se caracteriza por el desarrollo de la cultura Huarpa (Lumbreras 1974, 2000). Si bien se reconoce la importancia de esta cultura como la base local sobre la que luego se erigiría Huari, las investigaciones acerca de este período en el valle han sido escasas, y poco se conoce de la cultura Huarpa más allá del característico estilo cerámico a base del cual fue definida. Sólo se dispone actualmente de evidencias arqueológicas fragmentarias que no permiten determinar con precisión las características que presentaba la sociedad ayacuchana en estos tiempos. Se cree que este período de la prehistoria de Ayacucho se caracterizó por la presencia de unidades políticas de pequeña escala, quizás señoríos, representadas por conjuntos de sitios centrados en 
torno a uno o varios asentamientos principales (Isbell 2001; Lumbreras 2000; Schreiber 1992). Nawinpukyo habría formado, junto con Conchopata, Acuchimay y sitios menores, uno de estos enclaves, controlando el sur del valle de Ayacucho (Isbell 2001:114) (Figura 1).

La ocupación de la cima de Nawinpukyo en el período Intermedio Temprano tenía un carácter complejo, presentando varios sectores arquitectónicos netamente diferenciados (Figura 2). Parece claro que entre los siglos IV y VI d.C. el núcleo de la ocupación estaba constituido por la Plaza Este, un gran recinto de forma irregular que corona la cima de la colina, y que habría tenido primordialmente propósitos ceremoniales. La presencia de cerámica diagnóstica tanto en la superficie como en las excavaciones desarrolladas, justo al norte y sur de la plaza, indicaría que conjuntos de edificios Huarpa, quizás de naturaleza residencial, podrían haber existido en estos sectores, aunque nuestras investigaciones no revelaron restos arquitectónicos o evidencias primarias de ocupación. La construcción de edificios en estas áreas durante el Horizonte Medio podría haber resultado en el desmantelamiento de cualquier arquitectura más temprana. La presencia de cerámica Huarpa en superficie sobre un área rocosa a corta distancia al sudoeste de la plaza, y en la parte oeste de la cima de la colina (áreas no investigadas sistemáticamente) sugiere que podrían haber existido otros núcleos de ocupación, aunque sus características exactas permanecen desconocidos. Asimismo,

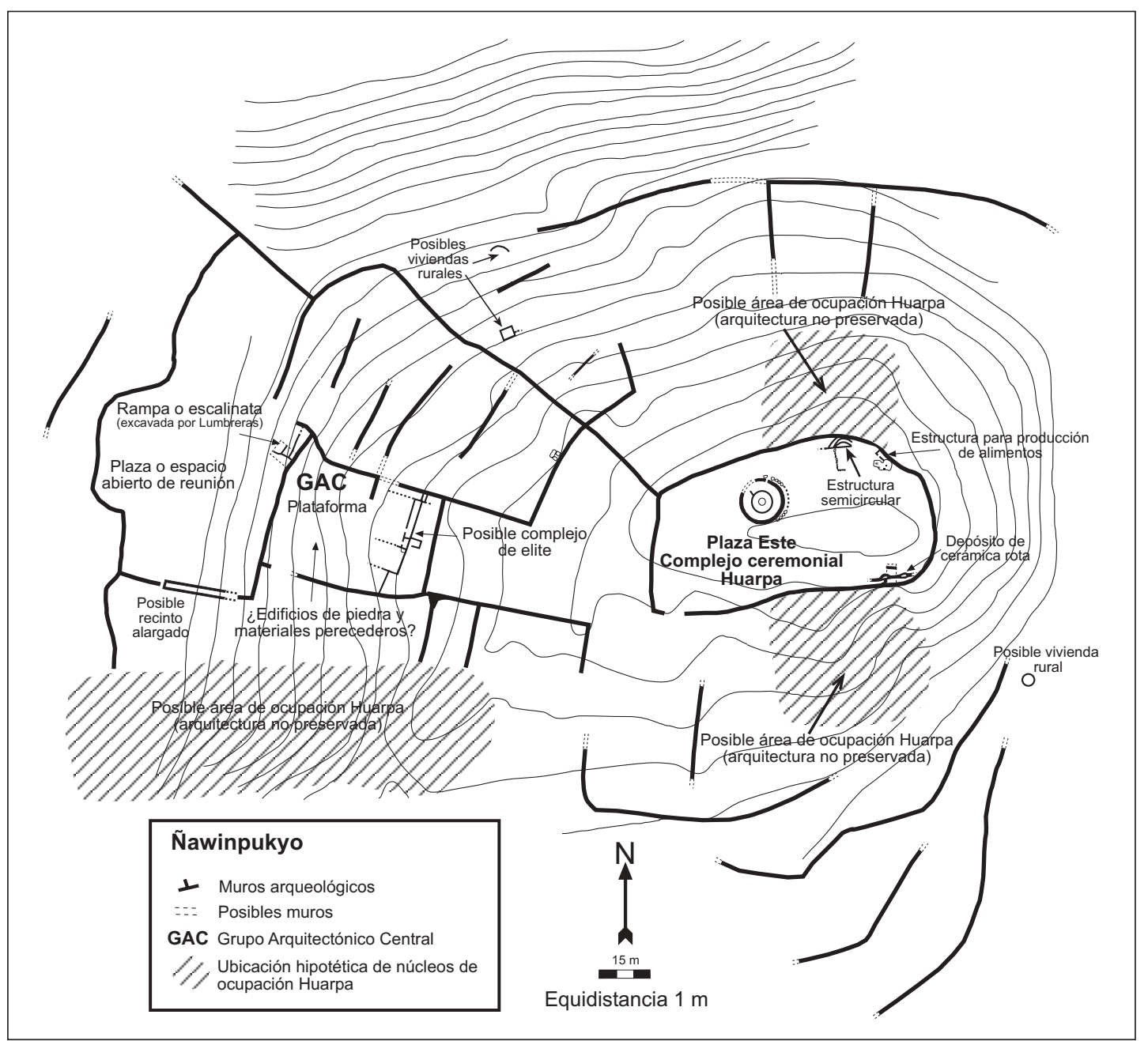

Figura 2. Principales componentes de Ñawinpukyo durante la ocupación Huarpa (período Intermedio Temprano). Nawinpukyo site plan showing its main components during the Huarpa occupation (Early Intermediate period). 
y tal como lo sugieren investigaciones anteriores (Cabrera 1998; Lumbreras 1974; Machaca 1997), existían núcleos de ocupación Huarpa en las partes bajas de la ladera oeste de la colina.

Un grupo importante de edificios, que posiblemente datan de finales del período Intermedio Temprano, fue identificado a unos $100 \mathrm{~m}$ al oeste de la plaza, en el sector que denominamos Grupo Arquitectónico Central (ver Lumbreras 1974:110) (Figura 2). Nuestras investigaciones en la parte noreste de este sector revelaron lo que parece haber sido parte de un complejo de elite, compuesto de galerías alargadas, cuartos y patios rectilíneos, con pisos y enlucidos blancos manufacturados con diatomita (roca sedimentaria abundante localmente) molida y compactada. Estas estructuras seguramente formaban parte de un conjunto mayor que se extendería hacia el oeste, pero que no pudo ser definido. No se encontraron evidencias de actividades domésticas o ceremoniales en ninguna de estas presuntas estructuras Huarpa.

\section{La Plaza Este}

La Plaza Este fue construida y utilizada probablemente durante la parte final del período Intermedio Temprano, a juzgar por la cerámica diagnóstica y por dos fechados radiocarbónicos (Tabla 1). Esta plaza amurallada mide aproximadamente $82 \mathrm{~m}$ de largo por $45 \mathrm{~m}$ de ancho, variando la altura de la pared perimetral entre $50 \mathrm{~cm}$ y $1 \mathrm{~m}$, dependiendo del estado de conservación de la misma. La plaza contiene una serie de estrcuturas que parecen haber servido fundamentalmente a un propósito ritual o ceremonial. Éstas incluyen una estructura semicircular en el lado norte de la plaza, un grupo de estructuras irregulares adheridas a la cara interna del muro perimetral de la plaza, y más notablemente, un edificio circular ubicado en la parte central al interior de la plaza (Figuras 2 y 3 ). Su posición única indica que esta estructura tuvo probablemente un rol preponderante en las actividades que tenían lugar en la plaza (Leoni 2004).

El edificio circular se compone de tres círculos concéntricos de piedra (Figura 3). El círculo exterior lo forma un muro de $1 \mathrm{~m}$ de ancho promedio, construido con dos hileras de piedras formando sus caras exteriores y piedras menores y tierra rellenando el interior; su diámetro interno oscila entre 11 y $11,5 \mathrm{~m}$. El círculo intermedio está formado por un muro de $60 \mathrm{~cm}$ de ancho, construido con doble hilera de piedras unidas con barro, con un diámetro interior de 5,6 m. Finalmente, un pequeño círculo construido con una sola hilera de piedras, de $1,9 \mathrm{~m}$ de diámetro interior y ancho promedio de $25 \mathrm{~cm}$, se encontraba justo al centro del edificio. Un segmento de muro radial que creaba una separación interna, evitando la libre circulación, se detectó en el cuadrante noroeste entre el círculo intermedio y el exterior. Finalmente, se detectaron varias piedras de gran tamaño, a aproximadamente $1 \mathrm{~m}$ del muro exterior, alineadas en forma evidente, aunque discontinua, constituyendo lo que podría haber sido un cuarto elemento circular concéntrico ${ }^{3}$.

El edificio circular sólo tiene una estrecha entrada, ubicada en su lado norte. Interesantemente, este acceso está perfectamente alineado con el nevado Rasuwillka, la montaña más alta visible desde el valle de Ayacucho y de gran importancia religiosa en el presente y posiblemente también en tiempos prehispánicos. Se establece así una conexión material directa entre el edificio y la montaña, pero también se produce un efecto visual cuidadosamen-

Tabla 1. Fechados radiocarbónicos, ocupación Huarpa (período Intermedio Temprano), Ñawinpukyo (Ayacucho, Perú). Radiocarbon dates from the Huarpa occupation (Early Intermediate Period) of Nawinpukyo (Ayacucho, Peru).

\begin{tabular}{lcccccc}
\hline$N^{\circ}$ de Muestra & $\begin{array}{c}\text { Fecha no } \\
\text { calibrada }\end{array}$ & $\begin{array}{c}\text { Fecha } \\
\text { calibrada } 1 \delta\end{array}$ & $\begin{array}{c}\text { Fecha } \\
\text { calibrada } 2 \delta\end{array}$ & $\mathrm{d}^{13} \mathrm{C}$ & Material & Proveniencia \\
\hline Beta-180665 & $1600 \pm 70$ & $\begin{array}{l}400-540 \text { d.C. } \\
\text { 320-620 d.C. }\end{array}$ & $260-290$ d.C. & -23.5 & Frijoles & $\begin{array}{c}\text { Plaza Este, estructura } \\
\text { quemados semicircular } \\
\text { (entre muros) }\end{array}$ \\
AA-46633 (*) & $1583 \pm 34$ & 425-537 d.C. & $408-560$ d.C. & -21.2 & Carbón & $\begin{array}{l}\text { Plaza Este, edificio } \\
\text { circular (bajo piso) }\end{array}$ \\
\hline
\end{tabular}

(*) Calibrada con programa CALIB 4.3 (Stuiver y Reimer 1993). 
te buscado, canalizando intencional e inevitablemente la percepción de las personas que abandonan el edificio directamente hacia la montaña sagrada (Figura 4), en lo que podría haber constituido una experiencia sensorial y religiosa muy significativa. De esta manera, se puede deducir inequívocamente que tanto el edificio como las actividades desarrolladas en él estaban directamente relacionados con el nevado.

Los contextos arqueológicos hallados dentro del edificio indican un posible uso ritual del mismo. No se encontraron materiales o asociaciones especiales en el elemento circular central ni en el espacio comprendido dentro del círculo intermedio, haciendo suponer que el interior de este edificio se habría mantenido cuidadosamente limpio. El único contex- to significativo está dado por una concentración de huesos de camélido desarticulados junto a la cara interna del muro circular intermedio (Figura 3). El espacio entre el círculo intermedio y el exterior, sin embargo, contenía un gran número de contextos arqueológicos. En primer lugar, en la parte sudeste del edificio se hallaron tres manos de moler sobre el piso del edificio, mientras que en la parte sudoeste se hallaron sobre el piso tres manos de moler asociadas con un batán. Estos hallazgos indican que algún tipo de molienda, quizás relacionado con la producción de comida y/o bebida, se realizaba dentro mismo del edificio.

Un hallazgo más significativo lo constituye la identificación de 23 concentraciones de huesos de fauna distribuidas por toda la parte norte y noreste

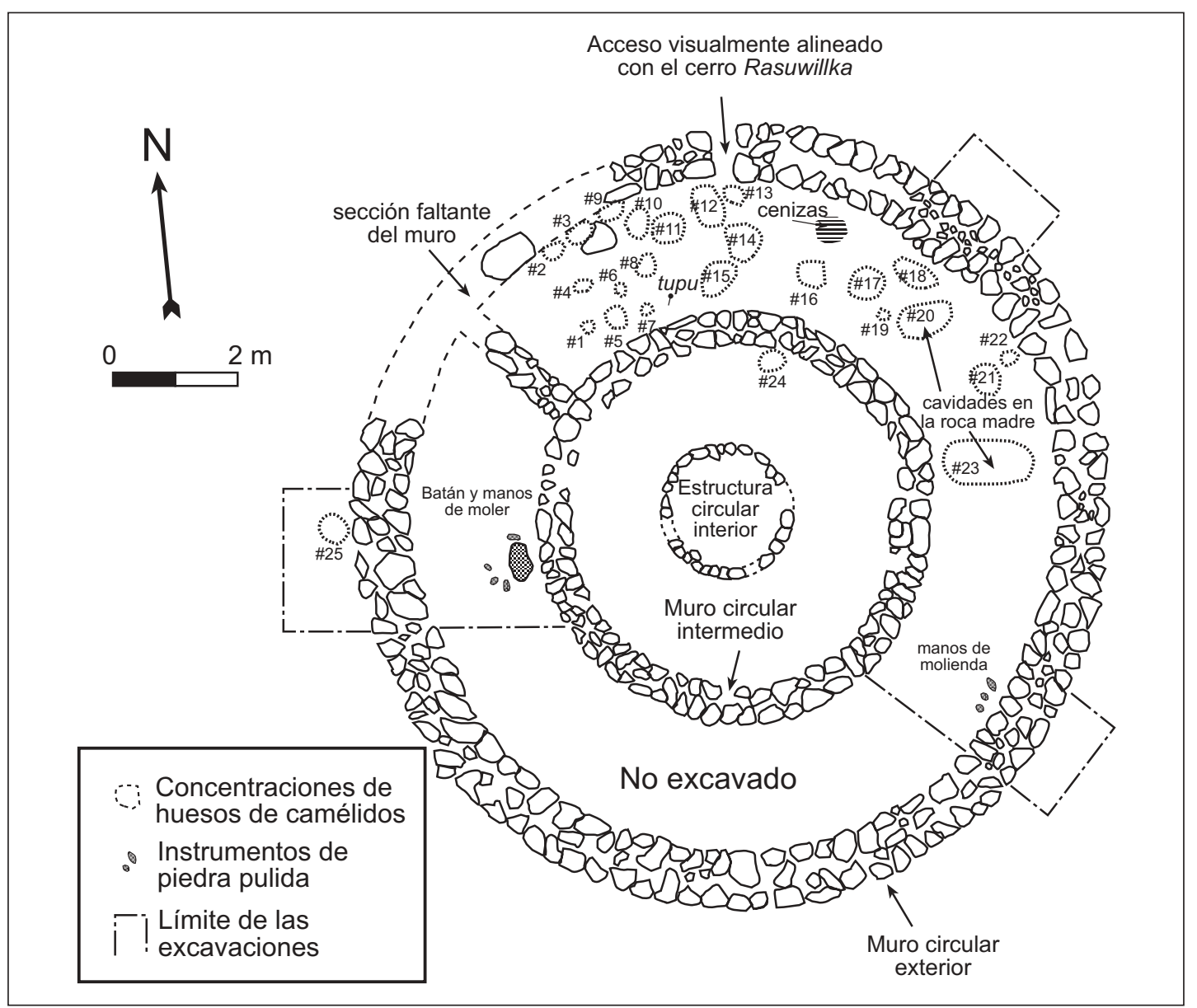

Figura 3. Planta del edificio ceremonial circular ubicado dentro de la Plaza Este en Nawinpukyo, mostrando los principales contextos arqueológicos excavados.

Plan of the circular ceremonial building located within the East Plaza, showing location of the main archaeological contexts excavated. 


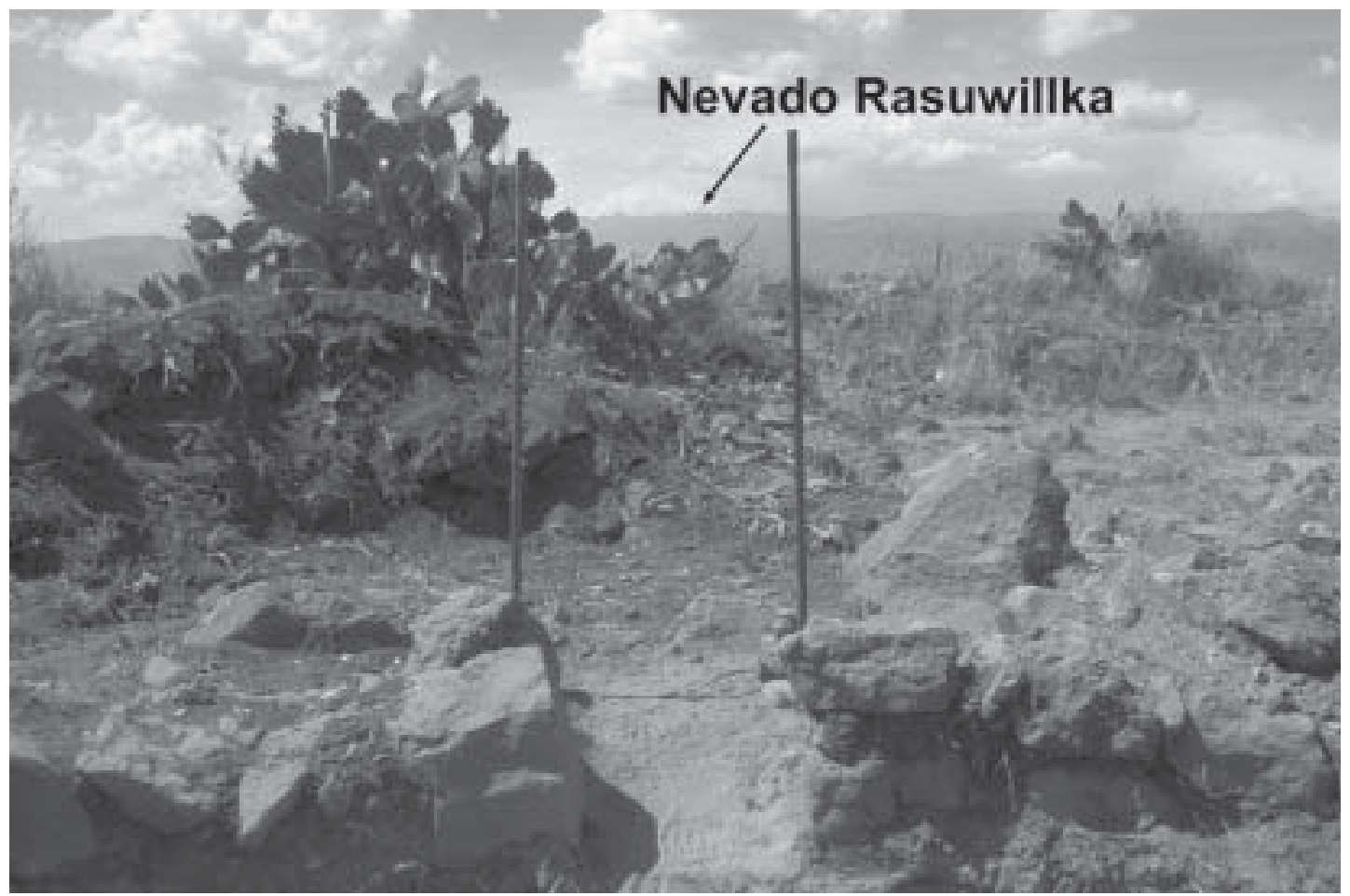

Figura 4. Vista del único acceso al edificio ceremonial circular en Nawinpukyo, alineado visualmente con nevado Rasuwillka. View of the circular ceremonial building's only doorway, showing its visual alignment with the Rasuwillka snow-capped mountain.

del espacio entre el muro intermedio y el exterior (Figuras 3 y 5). Los grupos constan de números variables de huesos desarticulados de camélidos: mientras algunos contienen los restos incompletos de varios animales, otros contienen sólo unos pocos huesos de un único animal (Tabla 2), y en uno de los grupos analizados se encontró un fragmento de cúbito humano ${ }^{4}$. Estos grupos de huesos fueron enterrados directamente en el piso de tierra del recinto y parecen haberse depositado en distintos momentos, a lo largo de la historia de uso del edificio como parte de las actividades desarrolladas dentro del mismo. Se han interpretado como el correlato de festines rituales, que incluían el sacrificio y consumo comunal de los camélidos (Leoni 2004).

El conjunto de estos hallazgos y la peculiar orientación de la entrada, junto con la forma del edificio, se conjugan para sugerir su posible función ceremonial. En efecto, en el área de Ayacucho son bien conocidos los edificios ceremoniales Huari en forma de "D" del Horizonte Medio (Cook 2001). Asimismo, se han descubierto edificios ceremoniales circulares en sitios como Huari (Isbell
2001:123), y más recientemente en Conchopata (Isbell y Cook 2002:291). Sin embargo, en ambos casos los edificios circulares se han atribuido tentativamente a momentos transicionales entre el período Intermedio Temprano y el Horizonte Medio. El edificio de Nawinpukyo, por el contrario, los antecede en el tiempo, construyéndose y usándose durante el período Intermedio Temprano. De esta manera, puede establecerse una secuencia ininterrumpida de desarrollo que llevó de los edificios ceremoniales circulares Huarpa a los templos en forma de "D" Huari, aunque las transformaciones ideológicas y en las prácticas religiosas que habrían acompañado a los cambios arquitectónicos permanecen en su mayor parte desconocidas.

Otro contexto interesante descubierto en la Plaza Este es un depósito de cerámica rota, localizado en el borde sudeste de la plaza, en una pequeña estructura $(1,5 \mathrm{~m}$ por $0,6 \mathrm{~m})$ semicircular de piedras junto al muro perimetral de la plaza. La cerámica rota (un total de 1.904 fragmentos, con un peso aproximado de $60 \mathrm{~kg}$ ), depositada en el interior de este receptáculo, corresponde a un gran 


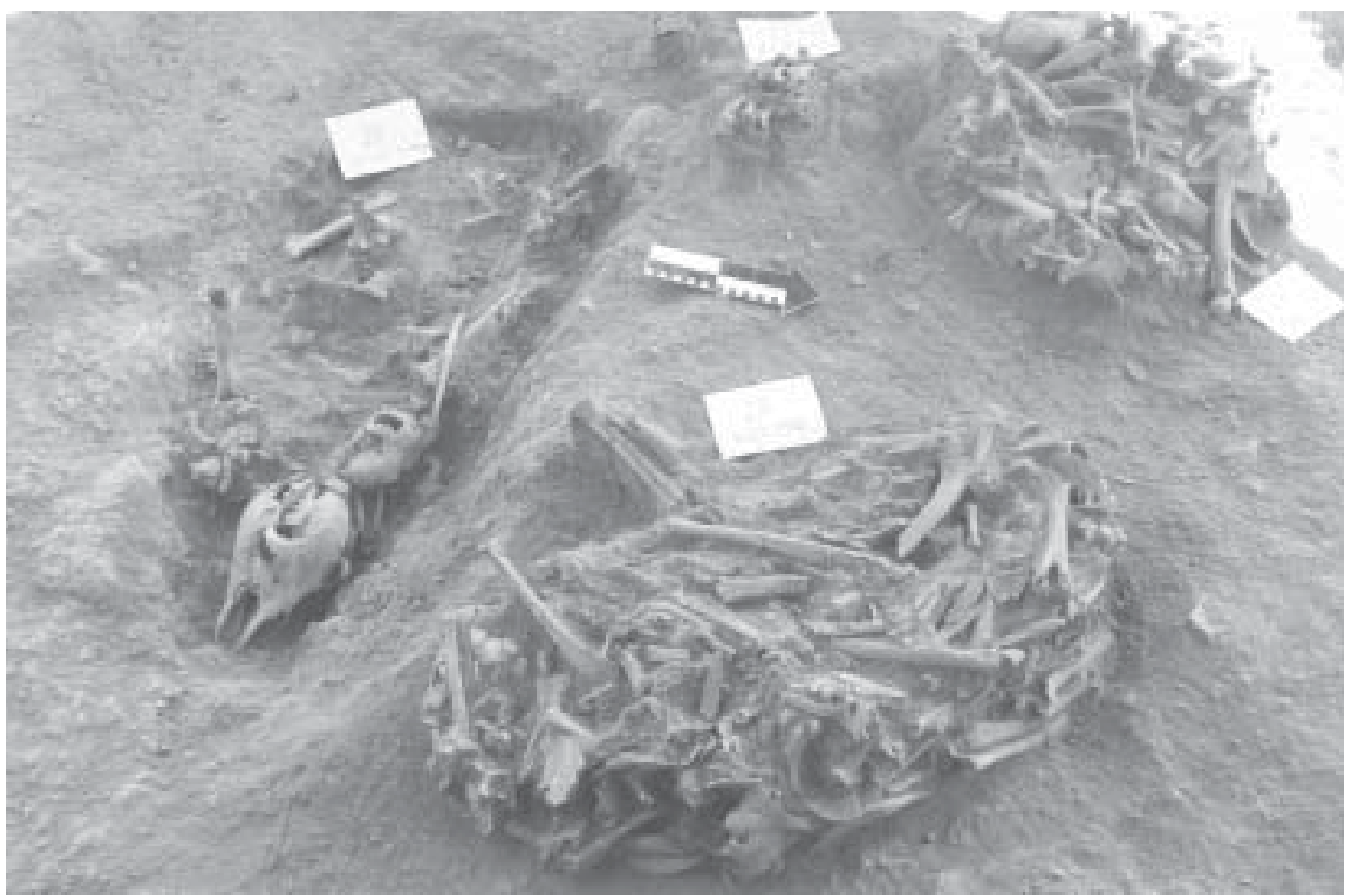

Figura 5. Concentraciones de huesos de camélidos halladas en el interior del edificio circular. Bones' concentrations found inside the circular building.

Tabla 2. Muestra analizada de grupos de huesos de fauna del edificio circular, Plaza Este, Nawinpukyo (según Rosenfeld 2002).

Sample of analyzed faunal bone concentrations from the circular building, East Plaza,

Nawinpukyo (after Rosenfeld 2002).

\begin{tabular}{lccll}
\hline $\begin{array}{l}\mathrm{N}^{\circ} \text { de } \\
\text { concentración }\end{array}$ & $\begin{array}{c}\mathrm{N}^{\circ} \text { especímenes } \\
\text { recuperados }\end{array}$ & $\begin{array}{c}\text { Total } \\
\text { NISP }\end{array}$ & $\begin{array}{c}\text { Total } \\
\text { MNE }\end{array}$ & MNI \\
\hline$\# 10$ & 935 & 296 & 153 & 5 (juveniles) \\
$\# 15$ & 174 & 87 & 62 & 3 (1 adulto, 1 juvenil, 1 tierno) * \\
$\# 18$ & 1156 & 408 & 230 & 9 (8 juveniles, 1 adulto) \\
$\# 20$ & 645 & 352 & 157 & 6 (4 juveniles, 1 tierno, 1 no determinado) ** \\
$\# 24$ & 200 & 113 & 44 & 1 (juvenil) \\
\hline
\end{tabular}

* Incluye un fragmento de cúbito humano.

** Incluye una alpaca de entre 9 y 12 meses de edad.

número de vasijas de diferentes formas y tamaños, tanto decoradas como no decoradas. El bajo índice de reconstructibilidad de las vasijas presentes indicaría que este depósito es de naturaleza secundaria y las condiciones de depositación parecen corresponder a un evento único. Las vasijas representadas incluyen principalmente cántaros grandes $(n=36)$, de diferentes formas y estilos, incluyendo ejemplares de la fina cerámica Cruz Pata y grandes vasijas de los estilos Kumunsenqa y Huarpa, y no decoradas. Cuencos $(n=21)$ y vasos $(n=2)$ también se encuentran representados en el conjunto, así como cuatro cucharas. La cuidadosa preparación de la estructura, así como la completa ausen- 
cia de otros tipos de materiales nos indican que este depósito no es un basural ordinario. La heterogénea composición formal del conjunto refleja una amplia gama de actividades relacionadas con la preparación y consumo de comida y bebida, y bien podría representar el correlato arqueológico de un festín ritual. Dado el gran número de cántaros presentes, este evento habría incluido un número significativo de participantes, tratándose quizás del mismo tipo de ritual que se presume originó las concentraciones de huesos de camélidos dentro del edificio circular.

\section{Interpretando la Plaza Este: Veneración de Montañas y Comunidad Local}

La evidencia recuperada de la Plaza Este parece corresponder al correlato material de actividades festivas a gran escala de índole religiosa. Estas prácticas habrían incluido el sacrificio y consumo de camélidos, y posterior entierro de sus restos en el espacio más sagrado dentro del complejo, así como el entierro ritual de las vasijas utilizadas en las ceremonias en estructuras especialmente preparadas. La llamativa relación del edificio circular con el nevado Rasuwillka, montaña de alto valor simbólico y religioso en la región, podría indicar que las ceremonias formaban parte de un culto a este cerro y que la veneración de montañas formaba parte central de la religión en Ayacucho en el período Intermedio Temprano. Estas ceremonias, a su vez, podrían haber jugado un rol importante en la integración social a nivel comunitario y supracomunitario.

Es interesante señalar que existen varias similitudes formales entre los eventos que presumiblemente produjeron los contextos arqueológicos en el edificio circular de Nawinpukyo y un sacrificio animal ritual dedicado al dios de una montaña descrito por Bolin (1998:53-57) en la comunidad puneña actual de Chillihuani (Cuzco, Perú). El uso de la analogía etnográfica para interpretar contextos arqueológicos ha sido objeto de intenso escrutinio en la arqueología contemporánea (Gould y Watson 1982; Stahl 1993; Wylie 1985), y los riesgos de su uso acrítico para la interpretación de la prehistoria andina han sido claramente señalados (Isbell 1997:303-308; Shimada 2003:90). Sin embargo, su valor, utilizado rigurosamente, en la exploración de procesos de cambio y continuidad en el pasado es generalmente reconocido (Stahl
1993:252; Wylie 1985:107). El uso que se hace aquí del caso etnográfico se enmarca en lo que Stahl (1993:236) denomina uso de la analogía como "modelo comparativo", enfoque que busca enfatizar tanto los puntos de convergencia como las diferencias entre el caso arqueológico y el análogo etnográfico para comprender mejor los eventos del pasado. Sobre la base de esto sugiero la posibilidad que los grupos de huesos de camélido halladas en Ñawinpukyo representen ritos propiciatorios, que apelaban al favor del cerro Rasuwillka para el éxito de las cosechas y la reproducción de los rebaños.

El caso etnográfico reportado por Bolin (1998:53-57) corresponde a un ritual familiar en el cual se sacrifica una llama negra. El sacrificio ocurre en el segundo día de la fiesta denominada Pukllay o carnaval, y forma parte de variados ritos que celebran la fertilidad, la procreación y la renovación de la vida. Según Bolin (1998:xi), esta fiesta tiene profundas raíces temporales en tiempos prehispánicos, a pesar de su asociación superficial con el carnaval posthispánico, y en ella se honra a las principales deidades andinas tradicionales (e.g. Pachamama, dioses de los cerros, dios del trueno). El animal es sacrificado por un curandero local contratado a tal efecto por la familia. La sangre del animal se recoge en un vaso, los órganos internos se comen, y la carne es distribuida entre los participantes para ser comida más tarde ${ }^{5}$. Significativamente, después que la carne ha sido consumida se recogen todos los huesos del animal y se los entierra juntos en el corral ceremonial de la familia. Los huesos faltantes son reemplazados por mazorcas de maíz para que el animal pueda resucitar en forma completa (Bolin 1998:54). El sacrificio se dedica al dios de montaña más importante en la región, Apu Ausangate, y se espera que el espíritu del animal muerto retorne a la montaña, alimentando al dios, quien, a su vez, retornará el animal al corral familiar a través de su renacimiento.

Varias semejanzas entre el caso arqueológico y el ritual etnográfico son evidentes. El entierro de los restos del animal en un espacio sagrado constituye tal vez el rasgo más notable. Las concentraciones de huesos animales en Nawinpukyo podrían, así, representar el entierro en suelo sagrado de animales sacrificados y consumidos en honor de deidades de montaña, tal como en el caso etnográfico de Chillihuani. Sin embargo, los animales hallados en Nawinpukyo no se encontraban completos, lo que sugiere que tal vez algunas partes se distri- 
buyeron para ser consumidos en otros lugares, se procesaron como charki y almacenaron para su consumo posterior, o simplemente se descartaron en otros lugares no detectados aún. Una diferencia de escala entre el caso arqueológico y el ritual documentado etnográficamente es muy evidente. Mientras que este último es un asunto menor, realizado en el ámbito de un grupo doméstico e implicando sólo a unos pocos individuos y a un especialista ritual, los eventos representados en la Plaza Este deben haber incluido a mucho más gente, algunas docenas por lo menos, a juzgar por el número de animales presentes en varios de los grupos de huesos. Por esto las ceremonias documentadas en Nawinpukyo habrían constituido eventos de mayor escala en los cuales habrían participado los miembros de la comunidad local y quizás de otras comunidades también.

En suma, ambos casos, arqueológico y etnográfico, parecen representar formas similares de prácticas de veneración de montañas andinas. Así, los contextos arqueológicos de Nawinpukyo podrían representar componentes prehispánicos de una tradición religiosa que manifiesta cierta continuidad hasta tiempos presentes. Sin embargo, esto no implica necesariamente que creencias y prácticas religiosas iguales a las registradas en Chillihuani existían ya en los siglos IV a VI d.C., y que los antiguos habitantes de Nawinpukyo percibieran a las montañas exactamente de la misma manera que los actuales pastores de la puna de Cuzco. A lo sumo, podemos inferir con alguna certeza que, por lo menos, algunos aspectos sobrenaturales asociados con las montañas formaban parte importante de la religión y cosmología Huarpa.

\section{Interpretando la Plaza Este: Ceremonias Religiosas e Integración Supracomunitaria}

Las ceremonias desarrolladas en el ámbito sagrado de la Plaza Este bien podrían haber funcionado como mecanismos integradores de la comunidad, agrupando a sus habitantes en prácticas comunes de manera periódica, tal vez anualmente. La comunidad misma habría sido celebrada y su identidad reafirmada en el proceso de honrar a los dioses de los cerros. La congregación periódica en el espacio sagrado de la plaza habría servido para afirmar la existencia y unidad de la comunidad local y su conexión con el territorio que ocupaba. Dado que se trataba de un marco religioso, la exis- tencia y reproducción de la comunidad, así como su orden interno, podrían haber recibido una legitimación divina. Conceptos acerca del orden social y cosmológico habrían hallado su expresión a través de estos eventos públicos. Pero las actividades rituales desarrolladas en la cima de Nawinpukyo podrían haber excedido el contexto puramente local, tal vez sirviendo también como mecanismo de integración supralocal y regional.

Si bien las evidencias arqueológicas no son aún concluyentes, los estudios etnográficos han mostrado repetidamente el carácter integrador de las ceremonias en que se venera a las montañas principales, que atraen a peregrinos de regiones enteras (Allen 2002:85; Bolin 1998; Poole 1991). Dada la reconocida importancia del nevado Rasuwillka como una poderosa deidad regional, existiría la posibilidad que la Plaza Este hubiera constituido un santuario que congregara al menos cierto grado de participación no local, tanto como foco de peregrinaje en sí mismo o como estación en una ruta de peregrinaje, que conducía a centros ceremoniales relacionados con Rasuwillka más importantes (y aún no descubiertos). El peregrinaje grupal en los Andes contemporáneos sirve para integrar espacios geográficos y sociales, articulando "un espacio social horizontal a través del espacio físico predominantemente vertical en el que los peregrinos se desplazan" [traducido por el autor] (Poole 1991:335). Es interesante que suelen existir paradas en lugares en donde las montañas sagradas o santuarios principales se hacen visibles, y rituales especiales se desarrollan en estos lugares. En este contexto, la ubicación de Ñawinpukyo adquiere gran significación. Localizado en el margen sur del valle de Ayacucho, controla la entrada al mismo desde el sur, suministrando una visión panorámica del valle y del nevado Rasuwillka. Para supuestos peregrinos prehispánicos, provenientes de áreas localizadas al sur de Ayacucho, Nawinpukyo y su centro ceremonial, podrían haber constituido una estación similar a las mencionadas en casos etnográficos, donde Rasuwillka se hacía visible. Si consideramos que las evidencias arqueológicas disponibles parecen indicar que no existía una centralización política marcada de la región en el período Intermedio Temprano, estas ceremonias y peregrinajes podrían haber tenido un importante rol como formas de integración social regional a través de la promoción de identidades comunes basadas en la religión. 


\section{Consideraciones Finales}

En suma, las recientes investigaciones arqueológicas en el sitio de Nawinpukyo parecen indicar que la veneración de montañas formaba parte central de la religión de los pueblos Huarpa del valle de Ayacucho durante el período Intermedio Temprano. Es interesante que, al menos formalmente, estas prácticas tendrían aspectos en común con prácticas y creencias similares reportadas etnográficamente, aunque resultaría prematuro todavía asegurar que esto se debe a la existencia de una larga continuidad cultural que mantuvo más o menos inalteradas un núcleo de creencias religiosas y cosmológicas. De hecho, no es posible determinar con certeza qué significaban las montañas para los antiguos ayacuchanos, más allá del obvio valor sobrenatural que parece innegablemente representado por los vestigios materiales.

En el caso concreto presentado, las ceremonias relacionadas con la veneración de montañas parecen haber constituido eventos públicos de gran importancia social y cultural para la comunidad local, y quizás haber ejercido también cierta influencia que excedía el ámbito puramente local, vinculando a diversas comunidades a base de un denominador ideológico común en tiempos en que no había en la zona una estructura política centralizada. Desgraciadamente, las evidencias arqueológicas no permiten aún determinar si existía un marcado control sociopolítico de estas ceremonias, tal vez a través del auspicio continuado de las mismas por un mismo grupo de descendencia o sector de la comunidad. Tampoco es posible determinar con certeza si las prácticas y el saber religioso asociados con estas ceremonias eran monopolizados por algún sector de especialistas. De haber sido así, estas ceremonias podrían haber constituido un ámbito de negociación de identidades sociales y políticas, $y$ tal vez una arena en la cual se desenvolvieron procesos de incremento de la desigualdad social que llevaron a la marcada estratificación social y centralización política asociados con el surgimiento de la sociedad Huari en el Horizonte Medio.

Si bien, como ya se señaló, Anders (1986) argumentó que las montañas sagradas constituyeron una parte muy importante de la cosmología Huari, al punto de basarse en ellas la organización territorial del estado, no encontramos en Nawinpukyo evidencias que indiquen una continuación clara de la veneración pública de los cerros durante el Ho- rizonte Medio. Si bien es cierto que el antiguo complejo ceremonial Huarpa fue preservado por los ocupantes Huari del sitio, implicando que el prestigio asociado con estos edificios y las prácticas desarrolladas en ellos permaneció en la memoria social de los siglos posteriores (Leoni 2004), no se han identificado en el lugar estructuras ceremoniales comunales comparables durante el Horizonte Medio. Asimismo, los típicos templos Huari en forma de "D" que se conocen en Ayacucho (ver Cook 2001) no muestran, en general, una asociación directa con el nevado Rasuwillka, como la descrita en este trabajo para el edificio Huarpa. Esto hace pensar que otras prácticas, tal vez en relación con nuevas concepciones religiosas y cosmológicas impulsadas por las elites de la poderosa sociedad Huari (p. ej., deidad de los báculos, cultos a los ancestros), quizás desplazaron el lugar central que ocupaba la veneración de montañas en Ayacucho en los siglos precedentes. Finalmente, una mejor comprensión del rol jugado por la veneración de montañas en el Ayacucho preincaico sólo se alcanzará cuando se expanda la muestra de edificios ceremoniales estudiados en el valle, y se investiguen sistemáticamente el área del nevado Rasuwillka y sus alrededores. Así podrá juzgarse mejor su rol tanto en las sociedades Huarpa y Huari como en otras sociedades ayacuchanas anteriores y posteriores.

Agradecimientos: El trabajo de campo en Nawinpukyo fue autorizado por el Instituto Nacional de Cultura de Perú (Resolución Directorial Nacional $\mathrm{N}^{\circ} 781,31$ de julio, 2001). La investigación se financió con una Doctoral Dissertation Improvement Grant (\#BCS-0105252) de la National Science Foundation. Una beca de Sigma-Xi, the Scientific Research Society ayudó a solventar en parte los fechados radiocarbónicos. El apoyo financiero de Binghamton University (SUNY) y Fundación Antorchas (Argentina) fue esencial para completar la tesis doctoral en la que este trabajo se basa. Agradezco a Aparicio Medina por la autorización para trabajar en su propiedad, y a los participantes del Proyecto Arqueológico Ñawinpukyo, en especial, Martha Cabrera (codirectora); Walter López, Teresa Limaylla, Manuel Lizárraga, Irela Vallejo, Ismael Mendoza, Edgar Alarcón, Diana Tamburini, y los trabajadores que participaron en las excavaciones. Un agradecimiento especial para William Isbell, José Ochatoma y Alberto Carbajal por su colaboración y asesoramiento constante a 
lo largo de todas las etapas del proyecto. Por último, agradezco las útiles observaciones de los eva- luadores anónimos de Chungara, Revista de Antropología Chilena, que revisaron este trabajo.

\section{Referencias Citadas}

Allen, C.J.

2002 The Hold Life Has: Coca and Cultural Identity in an Andean Community. Segunda edición. Smithsonian Institution Press, Washington D.C.

Anders, M.B.

1986 Dual Organization and Calendars Inferred from the Planned Site of Azángaro: Wari Administrative Strategies. Doctoral Dissertation. Cornell University, Ithaca.

Bastien, J.W.

1985 Mountain of the Condor: Metaphor and Ritual in an Andean Ayllu. Waveland Press, Prospect Heights.

Besom, J.T.

2000 Mummies, Mountains, and Immolations: Strategies for Unifying the Inka Empire's Southern Quarters. Doctoral Dissertation. Department of Anthropology, State University of New York, Binghamton.

Bolin, I.

1998 Rituals of Respect: The Secret of Survival in the High Peruvian Andes. University of Texas Press, Austin.

Cabrera Romero, M.

1998 Evaluación arqueológica en el complejo turístico de Ñawinpuquio. Informe presentado al Instituto Nacional de Cultura del Perú, Ayacucho.

Cook, A.G.

2001 Huari D-Shaped structures, sacrificial offerings, and divine rulership. En Ritual Sacrifice in Ancient Peru, editado por E. Benson y A. Cook, pp. 137-163. University of Texas Press, Austin.

Favre, $\mathrm{H}$.

1967 Tayta wamani. Le culte des montagnes dans le centre sud des Andes péruviennes. Publications des Annales de la Faculté des Lettres Aix-en-Provence 61:121-140.

Fernández Juárez, G.

1997 Entre la Repugnancia y la Seducción. Ofrendas Complejas en los Andes del Sur. Centro de Estudios Regionales Andinos Bartolomé de las Casas, Cusco.

Flannery, K., J. Marcus y R. Reynolds 1989 Flocks of the Wamani: A Study of Llama Herders on the Punas of Ayacucho, Peru. Academic Press, San Diego.

Glowacki, M. y M. Malpass

2003 Water, huacas, and ancestor worship: Traces of a sacred Wari landscape. Latin American Antiquity 14:431-448.

Gould, R.A. y P.J. Watson

1982 A Dialogue on the meaning and use of analogy in ethnoarchaeological reasoning. Journal of Anthropological Archaeology 1:355-381.

Guamán Poma de Ayala, F.

1980 [1615] Nueva Corónica y Buen Gobierno. Transcripción, prólogo, notas y cronología por Franklin Pease. Biblioteca Ayacucho, Caracas.

Isbell, B.J.

1978 To Defend Ourselves: Ecology and Ritual in an Andean Village. University of Texas Press, Austin.
Isbell, W.H.

1997 Mummies and Mortuary Monuments: A Postprocessual Prehistory of Central Andean Social Organization. University of Texas Press, Austin.

2001 Huari: crecimiento y desarrollo de la capital imperial. En Wari: Arte Precolombino Peruano, editado por P. Bazán y L. Millones, pp. 99-172. Fundación El Monte, Sevilla.

Isbell, W.H. y A.G. Cook

2002 A New Perspective on Conchopata and the Andean Middle Horizon. En Andean Archaeology II: Art, Landscape and Society, editado por H. Silverman y W.H. Isbell, pp. 249305. Kluwer Academic/Plenum Publishers, New York.

Kolata, A. y C. Ponce Sanginés

1992 Tiwanaku: the city at the center. En The Ancient Americas: Art from Sacred Landscapes, editado por R. Townsend, pp. 316-333. The Art Institute of Chicago, Chicago; Prestel Verlag, München.

Leoni, J.B.

2004 Ritual, Place, and Memory in the Construction of Community Identity: A Diachronic View from Ñawinpukyo (Ayacucho, Peru). Doctoral Dissertation, Department of Anthropology, State University of New York, Binghamton.

Lumbreras, L.G.

1974 Las Fundaciones de Huamanga. Hacia una Prehistoria de Ayacucho. Editorial Nueva Educación, Lima.

2000 Las Formas Históricas del Perú, Vol. 5: El Proceso de Regionalización. IFEA/Lluvia Editores, Lima.

Machaca Calle, G.

1997 Secuencia Cultural y Nuevas Evidencias de Formación Urbana en Ñawinpuquio. Tesis de Licenciatura, Universidad Nacional San Cristóbal de Huamanga, Ayacucho.

Martínez, G.

1983 Los dioses de los cerros en los Andes. Journal de la Société des Américanistes 69:85-115.

Morissette, J. y L. Racine

1973 La hiérarchie des wamani: essai sur la pensée classificatoire quechua. Recherches Amérindiennes au Québec 3:167-188.

Poole, D.A.

1991 Rituals of movement, rites of transformation: pilgrimage and dance in the highlands of Cuzco, Peru. En Pilgrimage in Latin America, editado por N. Ross Crumrine y A. Morinis, pp. 307-338. Greenwood Press, New York.

Reinhard, J.

1985 Sacred mountains: an ethnoarchaeological study of high Andean ruins. Mountain Research and Development 5:299-317.

1992 Interpreting the Nazca lines. En The Ancient Americas: Art from Sacred Landscapes, editado por R. Townsend, pp. 290-301. The Art Institute of Chicago, Chicago; Prestel Verlag, München.

Rosenfeld, S.

2002 Ñawinpukyo: análisis zooarqueológico. Manuscrito en posesión del autor. 
Schobinger, J. y M.C. Cerutti

2001 Arqueología de alta montaña en los Andes argentino. En Historia Argentina Prehispánica. Tomo II, editado por E.E. Berberián y A.E. Nielsen, pp. 523-559. Editorial Brujas, Córdoba.

Schreiber, K.J.

1992 Wari Imperialism in Middle Horizon Peru. Anthropological Papers of the Museum of Anthropology $\mathrm{N}^{\circ} 87$, University of Michigan, Ann Arbor.

Shimada, I.

2003 Revisión de Ritual Sacrifice in Ancient Peru, editado por E. Benson y A. Cook. Latin American Antiquity 14:89-91.
Silverman, H. y D. Proulx

2002 The Nasca. Blackwell Publishers, Malden and Oxford. Stahl, A.

1993 Concepts of time and approaches to analogical reasoning in historical perspective. American Antiquity 58:235-260.

Stuiver, M. y P.J. Reimer

1993 Extended ${ }^{14} \mathrm{C}$ database and revised CALIB radiocarbon calibration program. Radiocarbon 35:215-230.

Wylie, A.

1985 The reaction against analogy. Advances in Archaeological Method and Theory, vol. 8, editado por M. Schiffer, pp. 63-111. Academic Press, New York.

\section{Notas}

1 Se incluye bajo esta designación una extensa área geográfica que se extiende más allá de los límites del valle de Ayacucho propiamente dicho, incluyendo las provincias de Huanta, Huamanga, Víctor Fajardo, Lucanas y Cangallo en el Departamento de Ayacucho, y las provincias de Abancay y Andahuaylas en el Departamento de Apurímac.

2 El toponímico Rasuwillka (también escrito Raswillka o Razuwillka) se origina de la combinación de dos términos quechuas: rasu, que significa nieve o hielo, y que se usa normalmente para referir a una montaña nevada, y willka que generalmente significa nieto. Sin embargo, el término willka o villca (este ultimo utilizado por Guamán Poma (1980:193[1615]) al referirse a esta montaña) puede tener un significado más amplio. Así, Glowacki y Malpass (2003:436, Nota 7) señalan que villca también significa "abuelo," o "tatarabuelo," y por extensión "ancestro," pudiéndose, además, utilizarse en forma intercambiable con el término huaca, o persona, lugar u objeto que posee cualidades sobrenaturales. Por su parte, Bolin (1998:260) indica en su glosario de términos quechuas que tanto willka o villca significan "sagrado," con lo cual una posible traducción del toponímico resultaría "nevado sagrado".
3 El interior de la Plaza Este se usa actualmente con fines agrícolas. El uso del arado es probablemente responsable de que este alineamiento de piedras, así como otros que podrían haber existido en el centro de la plaza, no se haya preservado. La parte noroeste del edificio circular también parece haber sido afectada por este mismo proceso.

4 La presencia de un fragmento de cúbito humano entre los huesos de camélido podría sugerir la existencia de sacrificios humanos en Ayacucho en esta época. Los sacrificios humanos a las deidades andinas, y a los dioses de la montañas en particular, eran comunes en tiempos incaicos (Reinhard 1985; Schobinger y Cerutti 2001), y algunos estudios etnográficos han sugerido también la posibilidad de que sacrificios humanos propiciatorios a las montañas ocurrieran hasta tiempos relativamente recientes (Favre 1967:131). Sin embargo, la presencia de un único fragmento de hueso podría también responder a otras causas, como el uso de huesos humanos como objetos rituales o como parte de cultos a los ancestros.

5 Flannery et al. (1989:86) señalan que en rituales observados por ellos en la puna cercana a Ayacucho, en ocasiones parte de la carne del animal sacrificado es secada y transformada en charki para un consumo diferido. 